\title{
An Analysis of Coalition-Competition Pricing Strategies for Multi-Operator Mobile Traffic Offloading using Bi-objective Heuristics
}

\author{
Jone Consul ${ }^{1}$, Cristina Perfecto ${ }^{1}$, Miren Nekane Bilbao ${ }^{1}$, and Javier Del Ser ${ }^{1,2,3}$ \\ 1 University of the Basque Country UPV/EHU, 48013 Bilbao, Spain, \\ \{cristina.perfecto, javier.delser, nekane.bilbao\}@ehu.eus \\ 2 TECNALIA, E-48160 Derio, Spain \\ 3 Basque Center for Applied Mathematics (BCAM), 48009 Bilbao, Spain
}

\begin{abstract}
In a competitive market relationships between telecommunications operators serving simultaneously over a certain geographical area are diverse and motivated by very different business strategies and goals. Such relationships ultimately yield distinct pricing portfolios depending on the contractual affiliation of the user being served. Furthermore a key role in the last decade is the concept of tethering (connection sharing) which, when controlled by the operator, may help alleviating the consumption of network resources in densely populated scenarios. In this work we investigate the application of bi-objective heuristics for the design of Pareto-optimal network topologies leading to an optimal Pareto between the revenue of the incumbent operators in the scenario and the quality of service degradation experienced by the end users as a result of tethering. Based on computer simulation this work unveils that such a Pareto-optimal set of topologies is strongly determined by the market relationships between such operators.
\end{abstract}

Keywords: Traffic offloading; Pricing; Multiobjective; Competition; Coalition

\section{Introduction}

In the last years the telecommunication sector has witnessed an upsurge of the number of operators concurrently offering services over the same geographical area [1]. This sharp increase has been specially notable in the provision of mobile services, mainly due to the liberalization of this market in several economies and the decoupling between service deployment and infrastructure management, the latter introducing the role of the so-called virtual operator [2]. As such, virtual operators do not follow the conventional approach of acquiring a spectrum license to deploy a mobile network and offer their services, but rather enter the market by exploiting the infrastructure of incumbent operators to offer OverThe-Top (OTT) services [3]. The coexistence of these market stakeholders with traditional network operators lay the basis for a strongly competitive mobile market analyzed from different techno-economic perspectives [4-9]. 
In this context the appearance of multiple virtual players in the telecommunication market has grown at a significantly higher pace than the deployment of new access infrastructure. Content providers and virtual operators have come along with new mobile usage patterns by end users, who have increased their demanded resources from the network as a result of lower prices of terminal devices and the ubiquitous access to high-quality contents. According to Cisco [10] an exemplifying growth of $74 \%$ in the global mobile data traffic has been registered in 2015. The need for allocating resources to accommodate the challenging data explosion in mobile networks is what has pushed operators not only to share not only infrastructure in terms of core and radio access networks (as done by the aforementioned virtual operators), but also to explore opportunistic methods that involve their users themselves.

To this end, traffic offloading refers to the family of mechanisms aimed at minimizing the amount of information delivered over licensed communication resources which among other strategies can be accomplished by opportunistically resorting to local wireless communication technologies and dynamic content caching [11]. The criteria adopted in mobile traffic offloading can be very diverse, from the most straightforward policies (i.e. the maximization of the end-user satisfaction or the minimization of network operating expenses) to more elaborated schemes dealing with energy consumption, offload/upload persistence or the social centrality of the user along its predicted mobility trace [12]. Disregarding the criterion adopted to this end, the result is that traffic is shifted to opportunistically set inter-device networks.

In this paper we postulate that opportunistic traffic offloading can also provide interesting benefits when implemented between users of different mobile operators. In particular our work can be framed in the context of user incentives, by which users allow sharing their bandwidth for either offloading traffic of the operator to which they are subscribed, or providing service to subscribers of other operators. In this latter case incentives must be provided at two different levels of the scenario: between different operators and from operators to those subscribers from their client portfolio that should act as opportunistic relays and share their resources. This manuscript will explore how such incentive agreements impact on the Pareto trade-off between the quality of service delivered to end-users and the costs incurred to implement such policies. In particular we will resort to multi-objective heuristics to seek the set Pareto-optimal multi-hop network configurations -i.e. which nodes should be promoted to relays- under different incentive ratios among the operators. We will show that when operators ally together by agreeing low resource sharing fees, their overall benefit increases with respect to a competitive scenario with higher sharing fees. This increased benefit, however, yields a degraded quality of service to the end-user which, in a practical scenario, should be lower bounded in a per application basis. The simulations results obtained from different scenarios and incentive policies will be discussed so as to support these conclusions.

The reminder of this paper is structured as follows: Section 2 will formulate the optimization problem that models the offloading of traffic among operators, 
which will be efficiently solved by means of the solution encoding approach and heuristic solver described in Section 3 and subsections therein. The performance of the overall scheme is analyzed and discussed in Section 4 based on computer simulations. Section 5 ends the manuscript by drawing several conclusions.

\section{Problem Formulation}

This section presents key concepts and introduces the notation used throughout the rest of the paper. Let us suppose an area of dimensions $X_{\max } \times Y_{\max }$, where $N$ different operators offer their services so that the $n$-th operator - where $n \in$ $\{1, \ldots, N\}$ - has $M(n)$ clients, i.e. each operator serves a total of $M(n)$ users with $\sum_{n=1}^{N} M(n) \triangleq M$ denoting the total number of users existing in the area. Each operator will have a constant and circular coverage area with radius $R(n)$, whose limits fall within the previously defined area $X_{\max } \times Y_{\max }$. Such operators are connected to a backhaul station as shown in Figure 1. Nodes correspond to users' devices (e.g. smartphones, tablets, etc) located at coordinates $\left\{\left(x_{m}, y_{m}\right)\right\}_{m=0}^{M}$, each with its contracted services signed with operator $O(m) \in\{1, \ldots, N\}$.

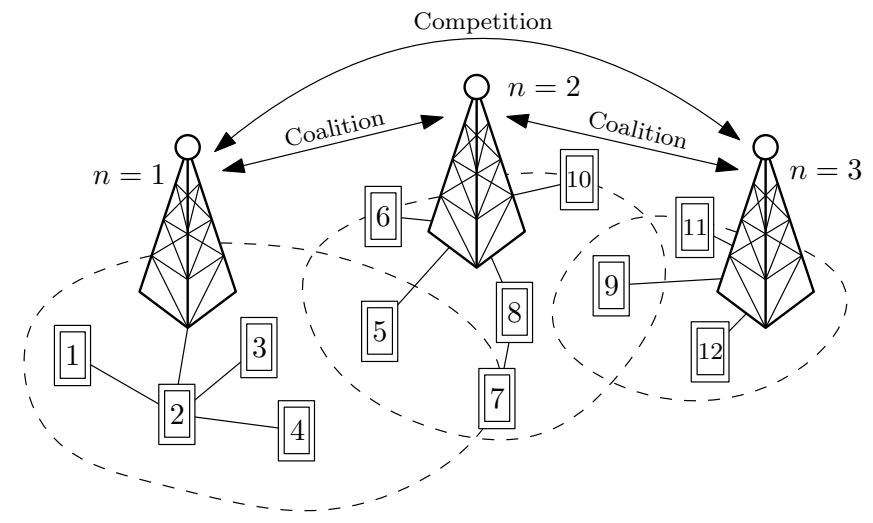

Fig. 1. Diagram of the scenario with $N=3$ operators and $M=12$ users. In this hypothesized situation user $n=2$ is sharing his/her connection with users $n \in\{1,3,4\}$, which may (or may not) be clients of the same operator.

User devices are assumed to be equipped with at least two wireless network interfaces such that one of them connects to Internet contents through the operator's cellular base station (BS), whereas the other resorts to short-range wireless protocols (e.g. WiFi or Bluetooth) to share its bandwidth with other users as a tethering interface. All devices operate in full duplex mode so as to be able to send data to the BS and receive data from other nearby nodes if tethering is enabled. Users under service can be connected to the backhaul through three different ways, each characterized by diverse requirements regarding the perceived quality of service and the cost for both the operator and the user/client: 
1. Via the operator contracted by the user: in this case the node will be connected directly to the operator with whom he/she has signed the contract. In this case the quality of service delivered to the user is expected to be contracted and ensured by the operator. For this rationale this direct connection policy yields the highest $\operatorname{cost} C_{*}$ for the operator among all the cases.

2. Via other third-party operator: in this second connection mode the node at hand will access the backhaul via an external operator with whom he/she has no signed contract. In this case a cost penalty must be taken into account to model the market relation between the operators in the management of each other's clients. A parameter $\alpha \triangleq C_{*} / C_{\circledast} \in[0,1]$ with $n \neq n^{\prime}$ is defined such that

- If $\alpha \approx 1$ the relationship between operators is a market coalition, i.e. the operational cost associated to the provision of services to any given user is approximately the same disregarding the operator with whom the user signed his/her contract (i.e. $C_{*} \approx C_{\circledast}$ ).

- If $\alpha \ll 1$ operators will be assumed to compete under a hostile market relationship, namely, it will be significantly more costly for an operator to serve its contracted clients through the premises of the other operator than through its own network equipment.

3. Via tethering: in this last option a node will connect to its BS through the tethered connection open by another user by virtue of the short-range wireless interface of his/her device. This case is expected to maximize the benefit of the operator and the users: operators can serve the user via tethering at a reduced cost $C_{T}$ modeling an incentive paid to the user sharing his/her connection, which is assumed to be lower than the cost $C_{*}$ incurred when serving the user directly. However, the relayed connection is assumed to degrade the quality of service experienced by the tethered user in the form of processing delays and/or reduced connection bandwidth. The relay node may represent a client from the same operator or instead, belong to any other provider.

The network operator $n \in\{1, \ldots, N\}$ obtains its benefit as the difference between the operational costs of providing service to its users and the monetary income yielded by their contracts $\left\{\Omega_{m}\right\}_{m=1}^{M}$. We simplify this cost as the sum of the costs associated to the price of the resources needed to establish the connection directly to the BS (collected in $C_{*}$ ), the price of serving a user through a connection of any other operator (resp. $C_{\circledast}$ ) and the cost of tethering a connection, in the form of an incentive $C_{T}$ to the client owning the tethering device. If we denote such a profit as $B(n)$, we have that

$$
B(n, \boldsymbol{\zeta}) \triangleq \sum_{m \in M(n)} \Omega_{m}-C_{m}^{t o t}(\boldsymbol{\zeta}),
$$

where the total cost per user $C_{m}^{t o t}(\boldsymbol{\zeta})$ is given by

$$
C_{m}^{t o t}(\boldsymbol{\zeta}) \triangleq C_{*} I(\zeta(m)=\mathrm{D})+C_{\circledast} I(\zeta(m)=0)+C_{T} I(\zeta(m)=\mathrm{T}),
$$

with $I(\cdot)$ as an auxiliary indicator function taking value 1 if its argument is true (and 0 otherwise). In the above formulae $\zeta(m) \in\{\mathrm{D}, \mathrm{O}, \mathrm{T}\}$ denotes the connection 
mode (DIRECT, OTHER, TETHERED) of user $m$ as per the description above. This defined budget will encompass the first optimization goal tackled in this study via the heuristic refinement of the vector of connection modes $\zeta \triangleq\{\zeta(m)\}_{m=1}^{M}$.

When the user at hand is tethered through the shared connection of any other user in the network, we will assume that the quality of service degrades in the form of a processing delay. The delay severity will be modeled as a numerical score whose value depends on 1) the number of hops from the user to the BS; 2 ) the number of simultaneously tethered users at the intermediate nodes that compose the path from the user to the BS. This numerical score $R(\boldsymbol{\zeta})$ will be set by the topology of the tree network modeling the connections between the underlying set of users and the BS deployed in the scenario, which in turn is given by the choice of connection modes $\zeta$ for the compounding nodes of the network. This tree is rooted on the backhaul, with $N$ first-level nodes representing the BS in the scenario, and intermediate/leaf nodes standing for the $M$ users.

While other progression models for the delay can be assumed instinctively, in this work we will compute $R(\boldsymbol{\zeta})$ as the average of the transmission slots to be waited by every node in the network in the best and worst cases. Assuming a round robin scheduling policy among users tethered at the same device, the best case stands for the case where node $m$ transmits directly through a multihop path to the BS without awaiting for any other's transmission. The latter (worst case) corresponds to the case when the node is scheduled for transmitting during the last transmission slot of every intermediate tethered set of users until the BS at hand. If $M_{l}^{m}(n)$ denotes the number of users at level $l$ in the subtree rooted on BS $n \in\{1, \ldots, N\}$ such that $\sum_{l} \sum_{m} M_{l}^{m}(n)=M(n)$ and all nodes in $M_{l}^{m}(n)$ share the same parent node as $m$; and $l(m) \in\left\{1, \ldots, L_{\max }^{n}\right\}$ (with $n=O(m)$ ) is the level at which user $m$ is located in the aforementioned subtree, this score particularized for BS $n$ will be given as

$$
R(n, \boldsymbol{\zeta})=\frac{1}{M(n)} \sum_{m \in M(n)} \frac{\left(R_{w c}^{m}(\boldsymbol{\zeta})+R_{b c}^{m}(\boldsymbol{\zeta})\right)}{2}=\frac{1}{M} \sum_{m=1}^{M} \frac{l(m)+M_{1}(n) \prod_{l^{\prime}=2}^{l(m)} M_{l^{\prime}}^{m}(n)}{2}
$$

namely, as the average between the number of hops between $m$ and its BS (best case) and the maximum number of scheduling slots that $m$ should await for transmission (worst case). Based on this definition, the problem tackled in this paper can be formulated as the search for a $K$-sized set of optimal connection modes $\left\{\boldsymbol{\zeta}_{k}^{\star}\right\}_{k=1}^{K}$ such that the Pareto trade-off between the operators' revenue and the quality of service experienced by the users is differently balanced, i.e.

$$
\left\{\boldsymbol{\zeta}^{\star}\right\}_{k=1}^{K}=\underset{\boldsymbol{\zeta} \in\{\mathrm{D}, 0, \mathrm{~T}\}^{M}}{\arg }[\max B(n, \boldsymbol{\zeta}), \min R(n, \boldsymbol{\zeta})],
$$

which can be read as the maximization of the operators' benefit and the minimization of the delay experimented by the users. From an intuitive perspective such optimization objectives are conflicting: in order to reduce operational expenditures, an operator will prefer that their users share as much bandwidth as possible via tethering and a favorable mechanism for incentives. Likewise, direct 
connections will ensure a high quality of service for the users, but will go against the business goal of the operator at hand. By solving the above optimization problem the decision maker commanding the operator of the network can easily trade one objective for the other as a function of the business priorities and the contractual requirements established at the time. In the next section we will describe the heuristic solver designed to efficiently deal with the above problem.

\section{Proposed Solver}

To solve the problem formulated in the previous section we will resort to a multi-objective version of the Harmony Search (HS) algorithm, a music-based meta-heuristic optimization method first presented in [13] as a result of the observation of the music improvisation procedure undertaken by musicians. When seeking a perfect harmony musicians rely on both their memory of notes played in the past and random pitch variations, which are emulated in a computer program much alike crossover and mutation processes in Evolution are mimicked in Evolutionary Algorithms. Notes played by the musicians represent the values of the optimization variables, which are iteratively refined by applying the aforementioned operators until a stop criterion is met (e.g. a maximum number of iterations or a steady convergence of the fitness values along successive iterations). The good performance scores obtained by this heuristic has been evinced in many application scenarios [14], with several prior contributions in the telecommunication domain [15-18].

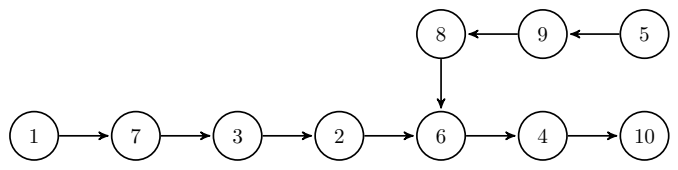

Fig. 2. Tree structure represented by the Dandelion string $\mathbf{C}=\{6,2,4,9,3,7,6,8\}$.

In order to represent numerically the solutions to the problem in Expression (3) (i.e. $\boldsymbol{\zeta}$ ) the Dandelion code will be used to represent the tree-like network topology that jointly represents nodes connected to the backhaul under the three connection modes $\{\mathrm{D}, \mathrm{O}, \mathrm{T}\}$ considered in this work. It is important to observe that by evolving this tree the connection mode is determined depending on which nodes result to be connected to each other. The Dandelion code is a bijective mapping between a tree network topology of $M+N+1$ nodes and an integer string with length $M+N-1$. This code has several properties in terms of inheritance and locality that make it suitable for tackling tree optimization problems by means of Evolutionary Algorithms [19], particularly in telecommunications [20-22]. A brief explanation of the encoding and decoding processes is now given:

- Dandelion encoding: Given a tree on $n$ nodes $T \in \Gamma_{n}$, usually in the form of an adjacency list or connectivity matrix, with $\Gamma_{n}$ denoting the set of possible trees interconnecting $n$ nodes: 
- Step 1: list intermediate nodes on the path from 1 to $n$ in $T$. Referring to the example tree given in Figure 2, these are nodes 7, 3, 2, 6 and 4.

- Step 2: find cycles in the list $\pi$ by searching for limit elements, namely, elements larger than any other to their right. Such elements for $\pi$ in the example list are 7, 6 and 4 and thus cycles are $(7),(3,2,6)$ and 4 .

- Step 3: the array $A_{C}$ for tree $T$ is constructed by filling its first row with elements $2,3,4, \ldots, n-1$ and adding cycle-related information into its second row, i.e.

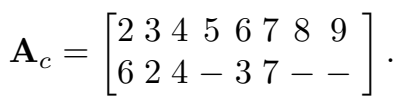

- Step 4: $C_{i+1}=\succ_{i}$ for every $i \in[2, n-1]$ and $\succ_{x}$ denoting x's successor relationship. Then, the Dandelion code $C$ corresponding to tree $T$ is given by the contents of bottom row of $A_{C}$.

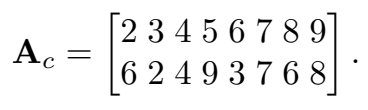

By looking at the complete $A_{C}$ representation of the example, the Dandelion code of the hypothesized tree $T$ is $\mathbf{C}=\{6,2,4,9,3,7,6,8\}$.

- Dandelion decoding: this procedure produces a tree $T \in \Gamma_{n}$ as follows:

- Step 1: a $2 \times n-2$ matrix $\mathbf{A}_{c}$ is built by inserting the integer set $\{2,3, \ldots, n-1\}$ in the first row and the elements of $C$ in the second row. For the exemplifying code $\mathbf{C}=\{6,2,4,9,3,7,6,8\}, \mathbf{A}_{c}$ is given as per (4)

- Step 2: define $f_{C}:[2, n-1] \rightarrow[1, n]$ such that $f_{C}(i)=C_{i}$ for each $i \in[2, n-1]$. Note that $f_{C}(i)$ corresponds to the $i$-th position $C_{i}$ of the code.

- Step 3: cycles associated to $f_{C}$ are computed as $\left\{Z_{1}, Z_{2}, \ldots, Z_{L}\right\}$. In the example 3 cycles, namely (2 63 ), (4) and (7), are obtained. Provided that $b_{l}$ denotes the maximum element in $Z_{l}$ (with $l \in\{1, \ldots, L\}$ ), cycles are then reordered such that $b_{l}$ is set as the rightmost element of $Z_{l}$, and that $b_{l}>b_{l^{\prime}}$ if $l<l^{\prime}$. In words, cycles are circularly shifted so that the largest element is the rightmost and sorted so that cycle maxima decreases from left to right. In the example this step yields $\left\{Z_{2}, Z_{1}\right\}=\{(7),(326),(4)\}$.

- Step 4: a list $\pi$ of the elements in $\left\{Z_{1}, Z_{2}, \ldots, Z_{L}\right\}$ is composed in the order they occur in the cycle list, from the first element of $Z_{1}$ to the last entry of $Z_{L}$, i.e. $\pi=\{(1)(7)(326)(4)(10)\}$.

- Step 5: the tree $T \in \Gamma_{n}$ corresponding to $C$ is constructed by arranging a set of $n$ isolated nodes labeled with the integers from 1 to $n$. A path from node 1 to node $n$ will be constructed by traversing the list $\pi$ from left to right. An edge will be included between nodes $i$ and $C_{i}$ for every $i \in\{2 \ldots, n-1\}$ not occurring in $\pi$. The tree corresponding to the Dandelion code $\mathbf{C}=\{6,2,4,9,3,7,6,8\}$ is the tree given in Figure 2 .

The compounding steps of the proposed bi-objective HS solver are as follows: 
1. Initialization: a pool of $\varphi$ solutions with lengths $M+N-1$ is initialized uniformly at random from the alphabet $\{2, \ldots, M+N\}$, which are evaluated according to the objectives as per (3).

2. Harmony improvisation: a new set of solutions is created from the previous set of harmonies by applying three stochastic operators: Harmony Memory Considering Rate (HMCR), Pitch Adjustment Rate (PAR) and Random Selection Rate (RSR), each driven by probabilistic parameters $P_{\mathrm{HMCR}}, P_{\mathrm{PAR}}$ and $P_{\mathrm{RSR}}$, respectively. We embrace the seminal definition of these operators proposed in [13] and extended in [23].

3. Fitness evaluation and memory update: the fitness values of the newly produced solutions is obtained and compared with those of the previous ones. As we deal with a bi-objective optimization problem a non-dominated sorting criterion based on dominance rank and crowding distance (similar to the one embedded in the well-known NSGA-II evolutionary solver [24]) is selected. Only the first $\varphi$ solutions in the list of harmonies ordered by front rank (first) and crowding distance (second) will be kept for the next iteration.

4. Termination: steps 2 and 3 are repeated until a number of iterations $\mathcal{I}$ set beforehand are completed.

\section{Experiments and Results}

In order to assess the performance of the proposed solver when addressing the bi-objective optimization problem stated in (3), two different scenarios have been created with two operators providing services over the same geographical area. The relation between both operators is defined by $\alpha$ as explained in Section 2. In all cases the area is $50 \times 50$ with a density of 20 nodes/users per operator. Values of the coverage radii $\{R(n)\}_{n=1}^{2}$ have been dynamically adjusted in order to provide service to at least $95 \%$ of the deployed users. Parameters of the HS solver are set to $\varphi=30, P_{\mathrm{HMCR}}=0.5, P_{\mathrm{PAR}}=P_{\mathrm{RSR}}=0.1$ and $\mathcal{I}=500$ iterations, with results averaged over 5 Monte Carlo experiments for each scenario. Incomes from service contracts are assumed to be $\Omega_{m}=1200 \forall m \in\{1, \ldots, M\}$, whereas costs are fixed to $C_{*}=1000$ (direct connection), $C_{T}=600$ (tethering incentive) and $C_{\circledast}=C_{*} / \alpha \in\{1000,10000\}$. (i.e. $\alpha \in\{1,0.1\}$ ). Regarding the latter it is important to note that $\alpha=1$ will emulate a coalition market agreement between operators, whereas $\alpha=0.1$ will correspond to a competitive market environment.

Table 1. Statistics of the obtained Pareto front estimations.

\begin{tabular}{|c|c|c|c|c|}
\hline & $\min B(n, \boldsymbol{\zeta})$ & $\max B(n, \boldsymbol{\zeta})$ & $\min R(n, \boldsymbol{\zeta})$ & $\max R(n, \boldsymbol{\zeta})$ \\
\hline Coalition $(\alpha=1)$ & 4000.00 & 8800.00 & 10.43 & 884.98 \\
\hline Competition $(\alpha=0.1)$ & 4400.00 & 8400.00 & 10.43 & 724.12 \\
\hline
\end{tabular}

The discussion focuses on Figure 3 and the statistics summarized in Table 1. As can be inferred from these obtained results, the income for the operators is lower for the case when both implement a hostile pricing policy - i.e. a low $\alpha-$ 
to provide services to external users. One would expect that this reduced profit would come along with a remarkable improvement in terms of quality of service for the end users (i.e. lower values of $R(n, \boldsymbol{\zeta})$ ). So do the obtained values for this metric, but differences with respect to the coalition case are only found in its maximum value. Therefore, from these results it can be concluded that for the simulated scenarios, a coalition scenario between service providers is favorable for increasing their average benefit whenever the degradation of the quality of service is admissible under the contractual conditions of their users.

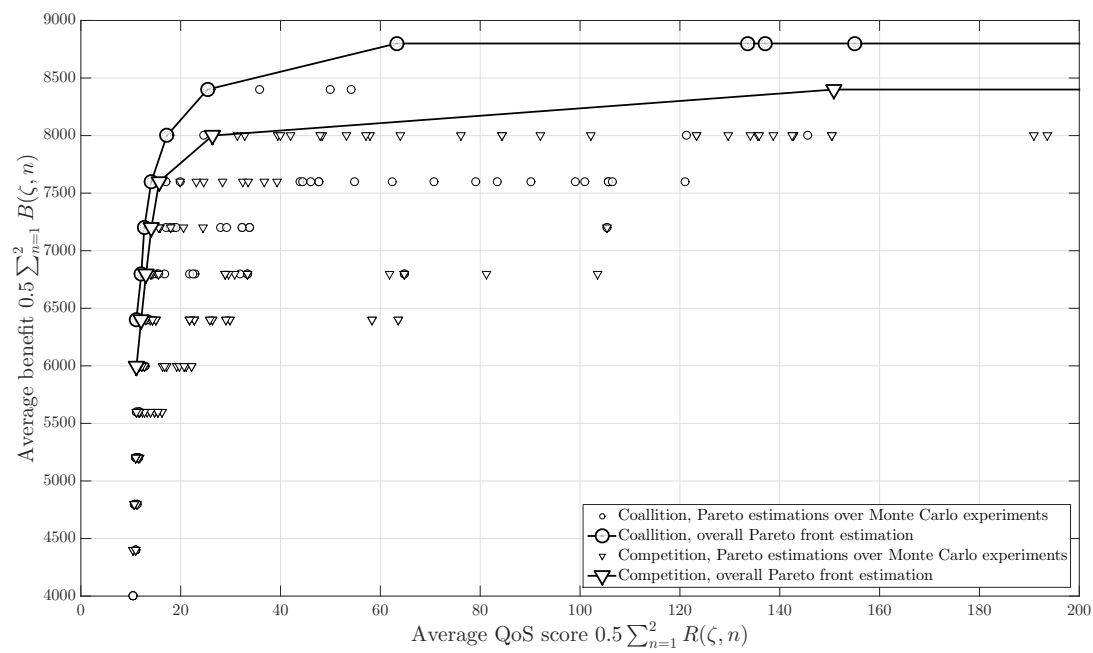

Fig. 3. Pareto Fronts trading average benefit for the quality of service offered to users under coalition and competition between operators.

\section{Concluding Remarks}

This manuscript has elaborated on analyzing the impact of different pricing agreements between service operators on the Pareto trade-off between their net income and the quality of service offered to their users. We have formulated this scenario as a bi-objective optimization problem, which relies on modeling the connection from users to the operators' network equipment as a tree graph that, in addition, accommodates the possibility of the operator to tether connections under incentive mechanisms. This graph is evolved towards Pareto-optimal configurations differently balancing quantitative metrics of the aforementioned optimization goals. The evolution is implemented efficiently by a heuristic solver that iteratively refines candidate solutions represented by means of the so-called Dandelion code, which possesses interesting features for evolving tree networks via crossover and mutation processes. Preliminary simulation results have evinced how such objectives behave when operators agree on a soft pricing policy for user roaming or, alternatively, impose hostile costs when processing users from any other counterpart. 


\section{Acknowledgments}

This work is supported by the Basque Government through the ELKARTEK program, in particular the BID3A (ref. KK-2015/0000080) and BID3ABI projects.

\section{References}

1. Maitland, C. F., Bauer, J. M., Westerveld, R. The European Market for Mobile Data: Evolving Value Chains and Industry Structures. Telecommunications Policy 26(9), 485-504 (2002)

2. Kiiski, A.: Impacts of MVNOs on Mobile Data Service Market. 17th European regional ITS conference (2006)

3. Greene, W., Lancaster, B.: Over the Top Services. LTC International Inc. (2007)

4. Gruber, H.: Competition and Innovation: The Diffusion of Mobile Telecommunications in Central and Eastern Europe. Information Economics and Policy 13(1), 19-34 (2001)

5. Kiiski, A., Hämmäinen, H.: Mobile Virtual Network Operator Strategies: Case Finland. ITS Conference (2004)

6. Smura, T., Kiiski, A., Hämmäinen, H.: Virtual Operators in the Mobile Industry: a Techno-Economic Analysis. NETNOMICS: Economic Research and Electronic Networking 8(1-2), 25-48 (2007)

7. Bertin, E., Noel, C., Michel, L.: A Few Myths about Telco and OTT Models. International Conference on Intelligence in Next Generation Networks (ICIN), 6-10 (2011)

8. Xu, X., Chen, R.: Competition, Cooperation, and Pricing: How Mobile Operators Respond to the Challenge of Over-The-Top. International Journal of Marketing Studies 7(6), 1-13 (2015)

9. Kibilda, J., Malandrino, F., DaSilva, L. A.: Incentives for Infrastructure Deployment by Over-The-Top Service Providers in a Mobile Network: A Cooperative Game Theory Model. IEEE International Conference on Communications, 1-6 (2016)

10. Cisco Visual Networking Index: Global Mobile Data Traffic Forecast Update, 20122017. Cisco, San Francisco, CA, USA. Available at http://www.cisco.com/en/US/solutions/collateral/ns341/ns525/ns537/ ns705/ns827/white_paper_c11-520862.html, accessed in November 2016.

11. Wang, X., Chen, M., Taleb, T., Ksentini, A., Leung, V. C.: Cache in the Air: Exploiting Content Caching and Delivery Techniques for 5G Systems. IEEE Communications Magazine 52(2), 131-139 (2014)

12. Siris, V. A., Kalyvas, D.: Enhancing Mobile Data Offloading with Mobility Prediction and Prefetching. ACM SIGMOBILE Mobile Computing and Communications Review 17(1), 22-29 (2013)

13. Geem, Z. W., Kim, J.-H., Loganathan, G. V.: A New Heuristic Optimization Algorithm: Harmony Search. Simulation 76(2), 60-68 (2001)

14. Manjarres, D., Landa-Torres, I., Gil-Lopez, S., Del Ser, J., Bilbao, M. N., SalcedoSanz, S., Geem, Z. W.: A Survey on Applications of the Harmony Search Algorithm. Engineering Applications of Artificial Intelligence 26(8), 1818-1831 (2013)

15. Forsati, R., Haghighat, A. T., Mahdavi, M.: Harmony Search based Algorithms for Bandwidth-Delay-constrained Least-Cost Multicast Routing. Computer Communications 31(10), 2505-2519 (2008) 
16. Del Ser, J., Bilbao, M. N., Gil-Lopez, S., Matinmikko, M., Salcedo-Sanz, S. Iterative Power and Subcarrier Allocation in Rate-constrained Orthogonal Multicarrier Downlink Systems based on Hybrid Harmony Search Heuristics. Engineering Applications of Artificial Intelligence 24(5), 748-756 (2011)

17. Del Ser, J., Matinmikko, M., Gil-Lopez, S., Mustonen, M.: Centralized and Distributed Spectrum Channel Assignment in Cognitive Wireless Networks: a Harmony Search Approach. Applied Soft Computing 12(2), 921-930 (2012)

18. Landa-Torres, I., Gil-Lopez, S., Del Ser, J., Salcedo-Sanz, S., Manjarres, D., Portilla-Figueras, J. A.: Efficient Citywide Planning of Open WiFi Access Networks using Novel Grouping Harmony Search Heuristics. Engineering Applications of Artificial Intelligence 26(3), 1124-1130 (2013)

19. Thompson, E., Paulden, T., Smith, D. K.: The Dandelion Code: A new Coding of Spanning trees for Genetic Algorithms. IEEE Transactions on Evolutionary Computation 11(1), 91-100 (2007)

20. Perfecto, C., Bilbao, M. N., Del Ser, J., Ferro, A., Salcedo-Sanz, S.: DandelionEncoded Harmony Search Heuristics for Opportunistic Traffic Offloading in Synthetically Modeled Mobile Networks. Harmony Search Algorithm 133-145 (2016)

21. Landa-Torres, I., Manjarres, D., Gil-Lopez, S., Del Ser, J., Salcedo-Sanz, S.: A Preliminary Approach to Near-optimal Multi-hop Capacitated Network Design using Grouping-Dandelion encoded Heuristics. IEEE International Workshop on Computer Aided Modeling and Design of Communication Links and Networks (CAMAD), 85-89 (2012)

22. Perez-Bellido, A. M., Salcedo-Sanz, S., Ortiz-Garcia, E. G., Portilla-Figueras, A., Naldi, M.: A Dandelion-encoded Evolutionary Algorithm for the Delay-Constrained Capacitated Minimum Spanning Tree Problem. Computer Communications 32(1), 154-158 (2009)

23. Mahdavi, M., Fesanghary, M., Damangir, E.: An Improved Harmony Search Algorithm for Solving Optimization Problems. Applied Mathematics and Computation 188(2), 1567-1579 (2007)

24. Deb, K., Pratap, A., Agarwal, S., Meyarivan, T. A. M. T.: A Fast and Elitist Multiobjective Genetic Algorithm: NSGA-II. IEEE Transactions on Evolutionary Computation 6(2), 182-197 (2002) 